\title{
Maxwell Superalgebra and Superparticles in Constant Gauge Backgrounds
}

\author{
Sotirios Bonanos, ${ }^{1}$ Joaquim Gomis, ${ }^{2,3}$ Kiyoshi Kamimura, ${ }^{4}$ and Jerzy Lukierski ${ }^{5}$ \\ ${ }^{1}$ Institute of Nuclear Physics, NCSR Demokritos, 15310 Aghia Paraskevi, Attiki, Greece \\ ${ }^{2}$ Departament ECM and ICCUB, Universitat de Barcelona, Diagonal 647, 08028 Barcelona, Spain \\ ${ }^{3}$ Facultad de Física, Universidad Católica de Chile, Santiago 22, Chile \\ ${ }^{4}$ Department of Physics, Toho University Funabashi 274-8510, Japan \\ ${ }^{5}$ Institute of Theoretical Physics, Wroclaw University, pl. Maxa Borna 9, 50-204 Wroclaw, Poland
}

(Received 3 December 2009; published 4 March 2010)

\begin{abstract}
We present the Maxwell superalgebra, an $N=1, D=4$ algebra with two Majorana supercharges, obtained as the minimal enlargement of a Poincaré superalgebra containing the Maxwell algebra as a subalgebra. The new superalgebra describes the supersymmetries of generalized $N=1, D=4$ superspace in the presence of a constant Abelian supersymmetric field strength background. Applying the techniques of nonlinear coset realization to the Maxwell supergroup we propose a new $\kappa$-invariant massless superparticle model providing a dynamical realization of the Maxwell superalgebra.
\end{abstract}

PACS numbers: 03.50.De, 04.40.Nr, 11.30.Ly, 12.60.Jv

Introduction.-Recently, after the discovery of the cosmic microwave background (CMB) and the mystery of dark energy [1], it is interesting to consider some field densities uniformly filling space-time. One such modification of empty Minkowski space is obtained by adding a constant electromagnetic (EM) field background, parametrized by the additional field degree of freedom $f_{\mu \nu}$. The presence of a constant EM field modifies the Poincaré symmetries into the so-called Maxwell symmetries [2-9]. The difference from the Poincare algebra consists in the de Sitter-like substitution (recall that dark energy is sometimes described by the addition of a cosmological term, or replacement of "empty" Minkowski space by de Sitter space)

$$
\left[P_{\mu}, P_{\nu}\right]=i Z_{\mu \nu}
$$

The additional tensorial generators $Z_{\mu \nu}$ are, however, Abelian and satisfy the relations

$$
\begin{gathered}
{\left[M_{\mu \nu}, Z_{\rho \tau}\right]=-i\left(\eta_{\nu \rho} Z_{\mu \tau}-\eta_{\nu \tau} Z_{\mu \rho}+\eta_{\mu \tau} Z_{\nu \rho}-\eta_{\mu \rho} Z_{\nu \tau}\right),} \\
{\left[P_{\mu}, Z_{\nu \rho}\right]=0, \quad\left[Z_{\mu \nu}, Z_{\rho \tau}\right]=0 .}
\end{gathered}
$$

The Bacry-Combe-Richard (BCR) algebra [2] is a subalgebra of the Maxwell algebra in which $Z_{\mu \nu}$ takes fixed numerical values. In the same way as the Poincaré algebra is the $R \rightarrow \infty$ limit ( $R=\mathrm{dS}$ radius) of de Sitter algebra, the Maxwell algebra $\mathcal{M}_{4}=\left(M_{\mu \nu}, P_{\mu}, Z_{\mu \nu}\right)$ given in (1) and (2) can be obtained by a suitable contraction of the de Sitter algebra $\left(\tilde{M}_{\mu \nu}, P_{\mu}\right)$ enlarged in a semisimple way by the Lorentz generators $M_{\mu \nu}$ (see also [8]). Performing the rescaling $P_{\mu} \rightarrow \alpha^{-1} P_{\mu}, \tilde{M}_{\mu \nu} \rightarrow \alpha^{-2} Z_{\mu \nu}, M_{\mu \nu} \rightarrow M_{\mu \nu}$ one obtains in the limit $\alpha \rightarrow 0$ the Maxwell algebra $\mathcal{M}_{4}$.

In order to interpret the Maxwell algebra and the corresponding Maxwell group, a Maxwell group-invariant particle model on the extended space-time $\left(x^{\mu}, \phi^{\mu \nu}\right)$ with the translations of $\phi^{\mu \nu}$, generated by $Z_{\mu \nu}$ has been studied [6-
9]. The interaction term described by a Maxwell-invariant one form introduces new tensor degrees of freedom $f_{\mu \nu}$ momenta conjugate to $\phi^{\mu \nu}$. In the equations of motion they play the role of a background EM field which is constant on-shell and leads to a closed, Maxwell-invariant two form.

The aim of this Letter is to obtain the supersymmetric extension of the Maxwell symmetries with new $N=1$ superMaxwell algebra and to investigate the corresponding superMaxwell-invariant massless superparticle model. (For massive superparticles one has to consider the $N=$ 2 supersymmetries in $D=4$ [10].) Analogously to the Maxwell case, one can introduce the generalized phase space with coordinates $\left(x^{\mu}, \theta^{\alpha}, \phi^{\mu \nu}, \phi^{\alpha}, \phi\right)$ and conjugate momenta $\left(p_{\mu}, \zeta_{\alpha}, f_{\mu \nu}, \tilde{\lambda}_{\alpha}, D\right)$. Since $\left(\phi^{\mu \nu}, \phi^{\alpha}, \phi\right)$ are cyclic coordinates the conjugate momenta $\left(f_{\mu \nu}, \tilde{\lambda}_{\alpha}, D\right)$ are constant on shell describing the constant Abelian SUSY $N=1$ gauge field background. In this way one gets the massless superparticle interacting with $x$ independent field strength superfield $W_{\alpha}(\theta)$

$$
W_{\alpha}(\theta)=i \tilde{\lambda}_{\alpha}-\frac{i}{2} f_{\mu \nu}\left(\bar{\theta} \gamma^{\mu \nu}\right)_{\alpha}-i D\left(\bar{\theta} \gamma_{5}\right)_{\alpha} .
$$

We see, therefore, that the superMaxwell symmetries describe the geometry of $N=1$ superspace $\left(x^{\mu}, \theta^{\alpha}\right)$ in the presence of constant SUSY gauge field background $\left(f_{\mu \nu}\right.$, $\left.\tilde{\lambda}_{\alpha}, D\right)$. It is also noted that the superparticle model is invariant under $\kappa$ transformations, which eliminate half of the Grassmann superspace coordinates $\theta^{\alpha}$.

Particle model with Maxwell symmetry.-To formulate a relativistic particle model, invariant under the Maxwell group, it is convenient to use the nonlinear coset realizations method [11]. The coset $G / H=$ Maxwell/Lorentz which we employ is parametrized as in [6-9], $g=$ $e^{i P_{\mu} x^{\mu}} e^{(i / 2) Z_{\mu \nu} \phi^{\mu \nu}}$. The basic Maurer-Cartan (MC) form is 


$$
\Omega=-i g^{-1} d g=P_{\mu} L^{\mu}+\frac{1}{2} Z_{\mu \nu} L_{Z}^{\mu \nu}+\frac{1}{2} M_{\mu \nu} L_{M}^{\mu \nu},
$$

where

$$
\begin{gathered}
L^{\mu}=d x^{\mu}, \quad L_{Z}^{\mu \nu}=d \phi^{\mu \nu}+\frac{1}{2}\left(x^{\mu} d x^{\nu}-x^{\nu} d x^{\mu}\right), \\
L_{M}^{\mu \nu}=0 .
\end{gathered}
$$

The particle action invariant under the Maxwell algebra (1) and (2) is described by the following Lagrangian:

$$
\mathcal{L}=\frac{\dot{x}_{\mu} \dot{x}^{\mu}}{2 e}-\frac{m^{2}}{2} e+\frac{1}{2} f_{\mu \nu} L_{Z}^{\mu \nu *},
$$

where $e$ is the einbein implementing the diffeomorphism invariance, $f_{\mu \nu}$ is a tensorial variable canonically conjugate to the new coordinates $\phi^{\mu \nu}$, and $L_{Z}^{\mu \nu *}$ is the pullback of $L_{Z}^{\mu \nu}$. In the proper time gauge, one obtains from (6) the equations of motion

$$
m \ddot{x}_{\mu}=f_{\mu \nu} \dot{x}^{\nu}, \quad \dot{f}_{\mu \nu}=0, \quad \dot{\phi}^{\mu \nu}=-\frac{1}{2}\left(x^{\mu} \dot{x}^{\nu}-x^{\nu} \dot{x}^{\mu}\right) .
$$

They describe the motion of a particle in an EM field $f_{\mu \nu}$, which is constant on shell. The EM potential is described by the one form $\mathcal{A}=\frac{1}{2} f_{\mu \nu} L_{Z}^{\mu \nu}$. In the closed two form field strength

$$
\mathcal{F}=d \mathcal{A}=\frac{1}{2} f_{\mu \nu} L^{\mu} \wedge L^{\nu}+\frac{1}{2} d f_{\mu \nu} \wedge L_{Z}^{\mu \nu}
$$

the second term vanishes on shell due to (7) and the field strength components are constants $f_{\mu \nu}$.

From Maxwell algebra to superMaxwell algebra.-We start with the following extension of the superPoincaré algebra in $D=4$ with Majorana supercharges $Q_{\alpha}(\alpha, \beta=$ $1,2,3,4)$

$$
\left\{Q_{\alpha}, Q_{\beta}\right\}=2\left(C \gamma^{\mu}\right)_{\alpha \beta} P_{\mu}, \quad\left[P_{\mu}, P_{\nu}\right]=i Z_{\mu \nu} .
$$

In order to verify the $(P, Q, Q)$ Jacobi identity, $P_{\mu}$ cannot commute with $Q_{\alpha}$ but requires a new Majorana charge $\Sigma_{\alpha}$ defined as

$$
\left[P_{\mu}, Q_{\alpha}\right]=-i \Sigma_{\beta}\left(\gamma_{\mu}\right)_{\alpha}^{\beta} .
$$

One can show from Jacobi identities that

$$
\left\{Q_{\alpha}, \Sigma_{\beta}\right\}=\frac{1}{2}\left(C \gamma^{\mu \nu}\right)_{\alpha \beta} Z_{\mu \nu} .
$$

$\Sigma_{\alpha}$, as well as $Q_{\alpha}$, transforms as a spinor under Lorentz transformations,

$$
\begin{aligned}
& {\left[M_{\rho \sigma}, Q_{\alpha}\right]=-\frac{i}{2}\left(Q \gamma_{\rho \sigma}\right)_{\alpha},} \\
& {\left[M_{\rho \sigma}, \Sigma_{\alpha}\right]=-\frac{i}{2}\left(\Sigma \gamma_{\rho \sigma}\right)_{\alpha} .}
\end{aligned}
$$

Together with relations (1) and (2) the superalgebra $G=$ $\left(M_{\mu \nu}, P_{\mu}, Z_{\mu \nu}, Q_{\alpha}, \Sigma_{\alpha}\right)$ is shown to close due to the gamma matrix identity $\left(C \gamma^{\mu}\right)_{(\alpha \beta}\left(C \gamma_{\mu}\right)_{\gamma \delta)}=0 \quad(\alpha \beta \gamma \delta$ symmetric sum) valid in $D=4$. $G$ defines the minimal Maxwell superalgebra containing the Maxwell algebra $\mathcal{M}_{4}$ as a subalgebra.
Consistently with the Jacobi relations one can also add a scalar central charge $B$ in (11) as

$$
\left\{Q_{\alpha}, \Sigma_{\beta}\right\}=\frac{1}{2}\left(C \gamma^{\mu \nu}\right)_{\alpha \beta} Z_{\mu \nu}+\left(C \gamma_{5}\right)_{\alpha \beta} B
$$

and obtain the centrally extended algebra $\tilde{\mathcal{G}}=$ $\left(M_{\mu \nu}, P_{\mu}, Z_{\mu \nu}, Q_{\alpha}, \Sigma_{\alpha}, B\right)$. It can be shown that the central charge $B$ corresponds to the constant mode of an auxiliary scalar in the "off shell" supersymmetric $U(1)$ gauge field theory.

Two Casimir operators of the Maxwell algebra obtained in $[2,3]$,

$$
\mathcal{C}_{2}=Z_{\mu \nu} Z^{\mu \nu}, \quad \mathcal{C}_{3}=Z_{\mu \nu} \tilde{Z}^{\mu \nu}, \quad\left(\tilde{Z}^{\mu \nu} \equiv \frac{1}{2} \epsilon^{\mu \nu \rho \sigma} Z_{\rho \sigma}\right)
$$

are also Casimir operators of the Maxwell superalgebra $G$, but the third mass Casimir operator requires a fermionic term

$$
\mathcal{C}=P^{2}+M_{\mu \nu} Z^{\mu \nu}+i \Sigma C^{-1} Q .
$$

For the centrally extended algebra $\tilde{G}$ the Casimir operator $\mathcal{C}$ ceases to commute with $Q$ and $\Sigma$. However, in the presence of an additional chiral symmetry charge $B_{5}$ satisfying

$$
\left[B_{5}, Q_{\alpha}\right]=-i\left(Q \gamma_{5}\right)_{\alpha}, \quad\left[B_{5}, \Sigma_{\alpha}\right]=i\left(\Sigma \gamma_{5}\right)_{\alpha},
$$

we can construct the extension of Casimir $\mathcal{C}$

$$
\tilde{\mathcal{C}}=P^{2}+M_{\mu \nu} Z^{\mu \nu}+i \Sigma C^{-1} Q-B_{5} B,
$$

which becomes a Casimir operator of the algebra $G_{5}=$ $\left(M_{\mu \nu}, P_{\mu}, Z_{\mu \nu}, Q_{\alpha}, \Sigma_{\alpha}, B, B_{5}\right)$. The super algebra $G_{5}$ will be realized in a massless particle model in the next section.

Massless superparticle model with Maxwell supersymmetry.-We construct a massless superparticle model using a nonlinear realization of the superMaxwell algebra $G_{5}$. The supergroup element $\tilde{g}$ is parametrized as

$$
\tilde{g}=e^{(i / 2) Z_{\mu \nu} \phi^{\mu \nu}} e^{i P_{\mu} x^{\mu}} e^{i \Sigma_{\alpha} \phi^{\alpha}} e^{i Q_{\alpha} \theta^{\alpha}} e^{i B \phi}
$$

using the supercoset $G / H=G_{5} /\left(M \times B_{5}\right)$ [12]. Here the chiral generator $B_{5}$ is in the unbroken subgroup because we construct a massless particle. The components of the MC form $\tilde{\Omega}=-i \tilde{g}^{-1} d \tilde{g}$ are

$$
\begin{aligned}
\tilde{L}^{\mu}= & d x^{\mu}+i\left(\bar{\theta} \gamma^{\mu} d \theta\right), \quad \tilde{L}^{\alpha}=d \theta^{\alpha}, \quad \tilde{L}_{M}^{\mu \nu}=0, \\
\tilde{L}_{Z}^{\mu \nu}= & d \phi^{\mu \nu}+i\left(\bar{\theta} \gamma^{\mu \nu}\right)_{\alpha} d \phi^{\alpha}+\frac{1}{2}\left(x^{\mu} d x^{\nu}-x^{\nu} d x^{\mu}\right) \\
& +\frac{i}{2}\left(\bar{\theta} \gamma^{\mu \nu} \gamma_{\rho} \theta\right)\left(d x^{\rho}+\frac{i}{6}\left(\bar{\theta} \gamma^{\rho} d \theta\right)\right), \\
\tilde{L}_{\Sigma}^{\alpha}= & d \phi^{\alpha}+\left(\gamma_{\rho} \theta\right)^{\alpha}\left(d x^{\rho}+\frac{i}{3}\left(\bar{\theta} \gamma^{\rho} d \theta\right)\right), \quad \tilde{L}^{5}=0, \\
\tilde{L}_{B}= & d \phi+i\left(\bar{\theta} \gamma_{5}\right)_{\alpha} d \phi^{\alpha}+\frac{i}{2}\left(\bar{\theta} \gamma_{5} \gamma_{\rho} \theta\right)\left(d x^{\rho}+\frac{i}{6}\left(\bar{\theta} \gamma^{\rho} d \theta\right)\right)
\end{aligned}
$$

and verify the corresponding MC equations 


$$
\begin{aligned}
d \tilde{L}^{\mu} & =i \overline{\tilde{L}} \gamma^{\mu} \tilde{L}-\tilde{L}_{M}^{\mu \nu} \tilde{L}_{\nu}, \quad d \tilde{L}_{M}^{\mu \nu}=-\tilde{L}_{M}^{\mu \rho} \eta_{\rho \sigma} \tilde{L}_{M}^{\sigma \nu}, \\
d \tilde{L}_{Z}^{\mu \nu} & =\tilde{L}^{\mu} \tilde{L}^{\nu}+i \overline{\tilde{L}} \gamma^{\mu \nu} \tilde{L}_{\Sigma}-\tilde{L}_{M}^{\mu \rho} \eta_{\rho \sigma} \tilde{L}_{Z}^{\sigma \nu}-\tilde{L}_{Z}^{\mu \rho} \eta_{\rho \sigma} \tilde{L}_{M}^{\sigma \nu}, \\
d \tilde{L}^{\alpha} & =\left(\gamma_{5} \tilde{L}\right)^{\alpha} \tilde{L}^{5}-\frac{1}{4} \tilde{L}_{M}^{\mu \nu}\left(\gamma_{\mu \nu} \tilde{L}\right)^{\alpha} \\
d \tilde{L}_{\Sigma}^{\alpha} & =\left(\gamma_{\mu} \tilde{L}\right)^{\alpha} \tilde{L}^{\mu}-\left(\gamma_{5} \tilde{L}_{\Sigma}\right)^{\alpha} \tilde{L}^{5}-\frac{1}{4} \tilde{L}_{M}^{\mu \nu}\left(\gamma_{\mu \nu} \tilde{L}_{\Sigma}\right)^{\alpha} \\
d \tilde{L}_{B} & =i \overline{\tilde{L}} \gamma_{5} \tilde{L}_{\Sigma}, \quad d \tilde{L}^{5}=0 .
\end{aligned}
$$

These MC equations provide a dual formulation of the superMaxwell algebra introduced in the previous section.

The massless superparticle action invariant under the superMaxwell group is

$$
\mathcal{L}=\frac{\pi_{\mu}^{2}}{2 e}+\mathcal{L}^{I *} ; \quad \mathcal{L}^{I}=\frac{1}{2} f_{\mu \nu} \tilde{L}_{Z}^{\mu \nu}+i \lambda_{\alpha} \tilde{L}_{\Sigma}^{\alpha}+D \tilde{L}_{B},
$$

where $\pi_{\mu}=\dot{x}_{\mu}+i \bar{\theta} \gamma_{\mu} \dot{\theta}$ is the pullback of $\tilde{L}_{\mu}$ to the world line and $e$ describes the einbein. Here $f_{\mu \nu}, \lambda_{\alpha}, D$ are dynamical variables transforming as Lorentz tensor, Majorana spinor and scalar, respectively. The interaction Lagrangian can be written explicitly as

$$
\mathcal{L}^{I^{*}}=\frac{1}{2} f_{\mu \nu} \dot{\phi}^{\mu \nu}+i \tilde{\lambda}_{\alpha} \dot{\phi}^{\alpha}+D \dot{\phi}+\pi^{\mu} A_{\mu}+\dot{\theta}^{\alpha} \tilde{A}_{\alpha},
$$

where

$$
\tilde{\lambda}_{\alpha}=\lambda_{\alpha}+D\left(\bar{\theta} \gamma_{5}\right)_{\alpha}+\frac{1}{2} f_{\mu \nu}\left(\bar{\theta} \gamma^{\mu \nu}\right)_{\alpha}
$$

and the $U(1)$ SUSY gauge potentials are

$$
\begin{aligned}
\tilde{A}_{\alpha}= & i\left(\bar{\theta} \gamma^{\mu}\right)_{\alpha}\left[-\frac{1}{2} f_{\mu \nu} x^{\nu}\right. \\
& \left.+i\left(\frac{2}{3} \tilde{\lambda}-\frac{1}{8} \bar{\theta} \gamma_{\rho \sigma} f^{\rho \sigma}-\frac{1}{4} D \bar{\theta} \gamma_{5}\right) \gamma_{\mu} \theta\right], \\
A_{\mu}= & -\frac{1}{2} f_{\mu \nu} x^{\nu}+i\left(\tilde{\lambda}-\frac{1}{4} \bar{\theta} \gamma_{\rho \sigma} f^{\rho \sigma}-\frac{1}{2} D \bar{\theta} \gamma_{5}\right) \gamma_{\mu} \theta .
\end{aligned}
$$

The variation of $\mathcal{L}$ with respect to $\left(\phi^{\mu \nu}, \phi^{\alpha}, \phi\right)$ gives

$$
\dot{f}_{\mu \nu}=\dot{\tilde{\lambda}}_{\alpha}=\dot{D}=0 ;
$$

i.e., the $U(1)$ superpotentials (24) are functions of the superspace coordinates $\left(x^{\mu}, \theta^{\alpha}\right)$ and the variables $\left(f_{\mu \nu}\right.$, $\left.\tilde{\lambda}_{\alpha}, D\right)$ which take constant values on shell. The variation of $\mathcal{L}$ with respect to $\left(f_{\mu \nu}, \tilde{\lambda}_{\alpha}, D\right)$ gives the equations for the variables $\left(\phi^{\mu \nu}, \phi^{\alpha}, \phi\right)$

$$
\left(\tilde{L}_{Z}^{\mu \nu}\right)^{*}=\left(\tilde{L}_{\Sigma}^{\alpha}\right)^{*}=\left(\tilde{L}_{B}\right)^{*}=0 .
$$

The variation of $\mathcal{L}$ with respect to $e$ puts the momenta $\pi_{\mu}$ on mass shell with vanishing mass

$$
\pi^{2}=0 .
$$

Finally, the variation of $\mathcal{L}$ with respect to $\left(x^{\mu}, \theta^{\alpha}\right)$ gives, using (24) and (25), the superparticle equations of motion in superspace,

$$
\begin{gathered}
\frac{d}{d \tau}\left(\frac{\pi_{\mu}}{e}\right)=\pi^{\nu} F_{\mu \nu}+\dot{\theta}^{\beta} F_{\mu \beta}, \\
2 i\left(\dot{\bar{\theta}} \gamma^{\mu}\right)_{\alpha}\left(\frac{\pi_{\mu}}{e}\right)=\pi^{\nu} F_{\nu \alpha},
\end{gathered}
$$

where the superfield strength using the differential operator $D_{\alpha}=\partial_{\alpha}+i\left(\bar{\theta} \gamma^{\mu}\right)_{\alpha} \partial_{\mu}$ are

$$
\begin{aligned}
F_{\mu \nu} & =\left(\partial_{\mu} A_{\nu}-\partial_{\nu} A_{\mu}\right)=f_{\mu \nu}, \\
F_{\mu \alpha} & =\left(\partial_{\mu} \tilde{A}_{\alpha}-D_{\alpha} A_{\mu}\right)=i\left(\lambda \gamma_{\mu}\right)_{\alpha},
\end{aligned}
$$

and the superspace constraints following from (24)

$$
F_{\alpha \beta}=\left(D_{\alpha} \tilde{A}_{\beta}+D_{\beta} \tilde{A}_{\alpha}\right)-2 i\left(C \gamma^{\mu}\right)_{\alpha \beta} A_{\mu}=0
$$

have been used in (29). The sector of our model covered by $\left(x^{\mu}, p_{\mu}, \theta^{\alpha}, \zeta_{\alpha}, f_{\mu \nu}, \tilde{\lambda}_{\alpha}, D\right)$ describes therefore a massless superparticle minimally coupled to the super $U(1)$ gauge field. Identifying the interaction term $\mathcal{L}^{I}=\mathcal{A}$ in (21) with the EM one-form superpotential, the two-superform field strength $\mathcal{F}=d \mathcal{A}$ is, after using the MC Eqs. (20),

$$
\mathcal{F}=d \mathcal{A}=\frac{1}{2} f_{\mu \nu} L^{\mu} L^{\nu}+i \lambda_{\alpha}\left(\gamma_{\mu} L\right)^{\alpha} L^{\mu}+\cdots,
$$

where the $\cdots$ terms are linear in the one forms $L_{B}, L_{\Sigma}^{\alpha}, L_{Z}^{\mu \nu}$ which vanish on shell. The field strength components are the ones given in (30) and (31).

Our model describes the coupling to a particular choice of $U(1)$ gauge superfield strength $W_{\alpha}(x, \theta)$ in (3), which satisfies the standard superspace constraints for the SUSY gauge theories [13],

$$
\begin{aligned}
F_{\alpha \beta} & =0, \quad F_{\mu \alpha}=W_{\beta}\left(\gamma_{\mu}\right)_{\alpha}^{\beta}, \\
D_{\alpha} W_{\beta} & =-\frac{i}{2}\left(C \gamma^{\mu \nu}\right)_{\alpha \beta} F_{\mu \nu}, \quad \partial_{\mu} W_{\beta}\left(\gamma^{\mu}\right)_{\alpha}^{\beta}=0 .
\end{aligned}
$$

It is known (see, e.g., [14]) that the coupling of the $N=1$ superparticle to the gauge superfield strength $W_{\alpha}(x, \theta)$ satisfying the constraints (33) leads to a $\kappa$-invariant interaction. Actually our system is not only invariant under the global Maxwell supersymmetries but also invariant under $\tau$ reparametrization and the $\kappa$ symmetries.

Conclusions.-In this Letter we found supersymmetric extensions of the Maxwell algebra and proposed a $\kappa$ invariant superparticle model (21) with the superMaxwell symmetries. It couples minimally to a constant $U(1)$ gauge superfield strength satisfying the superspace constraints [see (33)]. It gives a new geometric framework for a superspace filled with a uniform SUSY gauge field by generalizing the known nonsupersymmetric one with Maxwell symmetries. Because supersymmetries have critical importance in current fundamental interaction theories (e.g., string or $M$ theory), we hope such a generalization will be useful in this context, in particular, in the interpretation of fermionic backgrounds.

The superMaxwell algebra is realized if we regard the variables $\left(f_{\mu \nu}, \tilde{\lambda}_{\alpha}, D\right)$ as dynamical ones. In the 
Hamiltonian formulation of our model (21) they become the generators $\left(Z_{\mu \nu}, \Sigma_{\alpha}, B\right)$ of the superMaxwell symmetries. Note that by taking a fixed solution for $\left(f_{\mu \nu}, \tilde{\lambda}_{\alpha}, D\right)$ the superMaxwell symmetry is spontaneously broken to smaller ones similarly as in the bosonic case [2]. The evolution of the coordinates $\left(\phi^{\mu \nu}, \phi^{\alpha}, \phi\right)$ are described by Eq. (26) with their solutions determined by the trajectories in the "physical" subspace $\left(x_{\mu}, \theta_{\alpha}, f_{\mu \nu}, \tilde{\lambda}_{\alpha}, D\right)$. It will be interesting to find some physical interpretation for the new coordinates $\left(\phi^{\mu \nu}, \phi^{\alpha}, \phi\right)$ and their dynamical roles. For the bosonic Maxwell case it has been suggested [7] that $\phi^{\mu \nu}$ describes the magnetic moment of a distribution of charged particles with center-of-mass position $x^{\mu}$.

The superMaxwell algebra $\mathcal{G}$ introduced in this Letter is a minimal superextension of the Maxwell algebra. It can be considered as an enlargement of the Green algebra [15] by adding the tensorial central charges $Z_{\mu \nu}$. In the Green algebra the spinorial generators $\Sigma_{\alpha}$ are central [compare with (11)]. We have considered also its central extension $\tilde{\mathcal{G}}$ and the enlargement $G_{5}$ by means of the chiral generator $B_{5}$. The superMaxwell algebra $G$ can be embedded into larger superalgebras, in particular, in the known Bergshoeff-Sezgin (BS) p-brane algebra [16]. Thus one can introduce a corresponding BS-invariant superparticle model with the interaction Lagrangian generalizing (22) and gauge superpotentials $A_{\mu}^{\mathrm{BS}}, A_{\alpha}^{\mathrm{BS}}$ depending in a unique way on the BS supergroup coordinates. Using the coset with Lorentz stability group we find that the corresponding superfield strength $F^{\mathrm{BS}}$ 's do not satisfy the superspace constraints (33); i.e., the BS superparticle dynamics is not $\kappa$ symmetric. The origin of the noninvariance is the appearance of $Z_{\mu \nu}$ in the $\{Q, Q\}$ anticommutator resulting in $F_{\alpha \beta} \neq 0$ which violates the SUSY constraint (33) [cf. (32)]. We note also that Soroka and Soroka proposed in $[5,17]$ a nonstandard supersymmetrization of Maxwell algebra, without the translation generators in the basic anticommutator $\{Q, Q\}$; moreover in [17] there is presented some superextension of $k$-deformed Maxwell algebra $(k>$ 0 of [8]).

Our geometric scheme introduces additional degrees of freedom, describing uniform gauge field strengths in space and superspace leading to uniform constant energy density. These global degrees of freedom are dynamical; i.e., our model provides a framework in which the cosmological constant could be considered as a dynamical quantity. Recently, many papers propose new types of dynamics to explain the dark energy phenomenon (see, e.g., [18]) as well as the dynamical role of the cosmological constant (see, e.g., $[19,20])$. Because at present these issues are of fundamental importance, the developments in this Letter should find some important applications.

We acknowledge discussions with Jorge Alfaro. We also acknowledge financial support from projects FPA200766665-C02-01, 2009SGR502, Polish Ministry of Science and High Education grant NN202 318534, and Consolider CPANCSD2007-00042.

[1] For recent review see, for example, J. Frieman, M. Turner, and D. Huterer, Annu. Rev. Astron. Astrophys. 46, 385 (2008).

[2] H. Bacry, P. Combe, and J. L. Richard, Nuovo Cimento A 67, 267 (1970).

[3] R. Schrader, Fortschr. Phys. 20, 701 (1972).

[4] J. Beckers and V. Hussin, J. Math. Phys. (N.Y.) 24, 1295 (1983).

[5] D. V. Soroka and V. A. Soroka, Phys. Lett. B 607, 302 (2005).

[6] S. Bonanos and J. Gomis, J. Phys. A 42, 145206 (2009).

[7] S. Bonanos and J. Gomis, J. Phys. A 43, 015201 (2010).

[8] J. Gomis, K. Kamimura, and J. Lukierski, J. High Energy Phys. 08 (2009) 039.

[9] G. W. Gibbons, J. Gomis, and C. N. Pope, arXiv:0910.3220.

[10] J. A. de Azcarraga and J. Lukierski, Phys. Lett. B 113, 170 (1982).

[11] S. R. Coleman, J. Wess, and B. Zumino, Phys. Rev. 177, 2239 (1969); C. G. Callan, S. R. Coleman, J. Wess, and B. Zumino, Phys. Rev. 177, 2247 (1969).

[12] Some of the calculations with forms were done using the MATHEMATICA code for differential forms developed by S. Bonanos. See: "Graded Exterior Differential Calculus", http://www.inp.demokritos.gr/ sbonano/superEDC/.

[13] M. F. Sohnius, Phys. Rep. 128, 39 (1985).

[14] M. Rocek, W. Siegel, P. van Nieuwenhuizen, and A. E. van de Ven, Phys. Lett. B 227, 87 (1989).

[15] M. B. Green, Phys. Lett. B 223, 157 (1989).

[16] E. Bergshoeff and E. Sezgin, Phys. Lett. B 354, 256 (1995).

[17] D. V. Soroka and V. A. Soroka, Adv. High Energy Phys. 2009, 234147 (2009).

[18] E. J. Copeland, M. Sami, and S. Tsujikawa, Int. J. Mod. Phys. D 15, 1753 (2006).

[19] R. R. Caldwell and E. V. Linder, Phys. Rev. Lett. 95, 141301 (2005).

[20] S. Mukohyama and L. Randall, Phys. Rev. Lett. 92, 211302 (2004). 